\title{
The Median Frequency of the Surface EMG Power Spectrum in Relation to Motor Unit Firing and Action Potential Properties
}

\author{
H. J. Hermens, T. A. M. v. Bruggen, C. T. M. Baten, *W. L. C. Rutten, and \\ ${ }^{*}$ H. B. K. Boom \\ Rehabilitation Center "Het Roessingh," and *Biomedical Engineering Division, Department of Electrical \\ Engineering, University of Twente, Enschede, The Netherlands
}

\begin{abstract}
Summary: Three components determine the power spectrum of the surface EMG signal: the auto- and cross-power spectra of the firing processes and the power spectra of the motor unit action potential (MUAP). To clarify the relative contribution of these components to the median frequency (MF) of the power spectrum, a stochastic simulation model was used in which most input parameters [e.g., MUAP peak-peak time (PPT), mean interpulse interval time, and synchronization parameters] were described in terms of distribution functions. Simulation clearly predicts that MF is especially sensitive to variations in MUAP shape, the MUAP PPT, and synchronization. The influence of the firing process parameters was predicted to be marginal. To obtain values for the MUAP parameters, a needle-triggered averaging technique was used to gather surface MUAPs from the $\mathrm{m}$. biceps brachii. With use of these MUAPs as input for the model, it was found that intrasubject variability of MF is caused by variations in both MUAP PPT and MUAP shape, whereas intersubject variability in MF is caused primarily by variations in PPT. Key Words: Electromyography-Median frequency-Surface electrodes-Motor unit action potential-Synchronization-Simulation.
\end{abstract}

The noisy character of surface EMG records makes statistical signal analysis the most appropriate technique to obtain a quantitative description of the signal. In the frequency domain, the power spectral density function (power spectrum) is often used, one of its more explored parameters being the median frequency (MF). It has been shown that this parameter deviates from its normal value in a number of neuromuscular disorders $(2,9,17)$. MF is also

Accepted October 11, 1991.

Address correspondence and reprint requests to Dr. H. J. Hermens at Roessingh Research and Development, Roessinghbleekweg 33, 7522 AH Enschede, The Netherlands. a broadly accepted parameter to monitor muscle fatigue (6). Therefore, it has been applied in clinical $(24,33)$ as well as in more basic $(18,32)$ studies of fatigue. The use of special hardware makes it possible to monitor MF even on-line (15), which seems especially useful in ergonomic studies. Recently it was shown that the MF can also be used to identify recruitment control strategies (26).

Model studies have linked motor unit characteristics, like the firing process statistics and the motor unit action potential (MUAP), to properties of the power spectrum. A usual approach is to model single motor unit firing as a point process and an MUAP train as the convolution of this point process 
with a particular MUAP function (e.g., 5,13,22,30). In the frequency domain, this implies multiplication of the power spectra of the firing process and the MUAP. When more than one MUAP train is considered, the total power spectrum equals the sum of the power spectra of the individual trains only if there is no cross correlation between them, i.e., if there is no synchronization between the MUAP trains. In case of synchronization, the resulting cross-power spectra have to be taken into account.

There are thus three components that determine the power spectrum of the surface EMG signal: the auto- and cross-power spectra of the firing processes and the MUAP power spectra. The aim of this study is to clarify the relative contribution of these components to the MF of the surface EMG power spectrum. For this purpose a stochastic simulation model has been developed, tested, and used, in which most input parameters are described in terms of distribution functions to account for their natural variability.

To draw realistic conclusions, it was essential to use realistic values of the input parameters during simulation. Statistical properties of the firing process of individual motor units are described in the literature $(4,7,21)$. Hardly any experimental data could be found considering synchronization. In the theoretical studies on synchronization, different methods were used to link synchronized MUAP trains. Both constant (13) and random (31) delays between the corresponding firing moments in the different trains have been used, as well as the grouping of firing moments in bursts (1). Only Weytjens and van Steenberghe (31) based their method on experimental findings (11). This makes their model of synchronization the most appropriate to use.

Also no quantitative data were found describing the MUAP properties as observed with surface electrodes. Therefore, it was decided to obtain these data by experiment. A needle-triggered averaging method was applied to extract MUAP data from the interference EMG pattern of healthy subjects.

\section{METHODS}

\section{Theoretical Backgrounds}

An MUAP train can be described as the output of a linear time-invariant filter with a sequence of Dirac $\delta$-functions as input $(5,13,19,22,30)$ according to

$$
y(t)=f(t) * m(t)
$$

in which

$$
f(t)=\Sigma \delta\left(t-t_{i}\right)
$$

where $m(t)$ denotes the MUAP as observed by the surface electrode and $f(t)$ is a sequence of Dirac $\delta$-functions marking the points of time the MUAP occurs in the MUAP train. The asterisk (*) stands for convolution. We regard the surface EMG signal as

$$
e(t)=\sum_{i=1}^{n} y_{i}(t)
$$

where $n$ is the number of MUAP trains. Using Eqs. 1 and 3 it can be derived that the power spectrum $\operatorname{Se}(\omega)$ is given by $(21,31)$

$$
\begin{aligned}
S e(\omega)= & \sum_{i=1}^{n} S f_{i i}(\omega) \cdot\left|M_{i}(\omega)\right|^{2} \\
& +\sum_{\substack{i=1 \\
i \neq j}}^{n} \sum_{\substack{j=1 \\
i \neq j}}^{n} S f_{i j}(\omega) \cdot\left[M_{i}(\omega) \cdot M_{j}^{*}(\omega)\right]
\end{aligned}
$$

where $S f_{i i}(\omega)$ is the power spectrum of the firing process of train $i, S f_{i j}(\omega)$ the cross-power spectrum of the firing processes of train $i$ and train $j, M_{i}(\omega)$ is the Fourier transform of the impulse response of filter $i$, and $M_{i}^{*}(\omega)$ is the complex conjugate of $M_{i}(\omega)$.

The first term on the right represents the contributions of the individual trains if they were independent. The second term is due to correlation between the firing processes of different MUAP trains; in absence of correlation, this term is zero.

Several authors $(13,19,29)$ have investigated the power spectrum of the firing process of individual MUAP trains $[S f(\omega)]$. They found that peaks occurred in the power spectrum at a frequency corresponding to the mean firing rate and, with decreasing power density, at its harmonics. At frequencies several times higher than the mean firing rate, the power density became a constant value. The power density at the center of the peaks strongly depended on the regularity of firing. The peaks broadened and became lower when the regularity of firing decreased. This was also shown by De Luca (6) who 
calculated the Fourier transform of experimentally obtained interpulse interval (IPI) data directly.

Weytjens and van Steenberghe (31) investigated the cross-power terms in Eq. 4, modeling the time difference between corresponding action potentials in two MUAP trains as a Gaussian variable with zero mean. They found that synchronization resulted in an absolute increase of power at low frequencies, the size of this effect being dependent on the variance of the Gaussian variable.

The aim of this article is to contribute to a better understanding of the sensitivity of MF for changes in the terms in Eq. 4, when the parameters that determine these terms are varied within their natural range. Simulation was used to facilitate the implementation of the distribution functions that describe the variability of the input parameters.

\section{The Simulation Model}

Figure 1 shows the basic concepts of the model. The EMG signal was obtained by addition of a number $(N)$ of MUAP trains. In calculating each train, we distinguished three stages. First the MUAP was calculated, then the firing instants were determined. Finally generation of the MUAP at the firing instants created a MUAP train.

\section{MUAP Generation}

Each MUAP was described with three parameters: an MUAP shape normalized with respect to amplitude and duration, an amplitude, and a duration (see Fig. 1). The MUAP shapes were stored in a library that could contain up to 16 different shapes. During simulation each MUAP shape was assigned to a given percentage of the total number of MUAP trains.

The peak-peak voltage (PPV) of the biphasic MUAP was defined as the voltage (difference) between the positive and negative peak of the MUAP. The duration of the MUAP was parameterized by the peak-peak time (PPT), i.e., the time difference between the occurrence of the two peak values. All MUAP shapes in the library had equal (unity) values of PPT and PPV. During simulation, actual values of PPV and PPT were drawn from two independent Gaussian distribution functions (mean value $\mu_{V}$ and $\mu_{T}$; standard deviation $\sigma_{V}$ and $\sigma_{T}$ ). These values were used to multiply and resample the MUAP shapes. The MUAP properties were as-

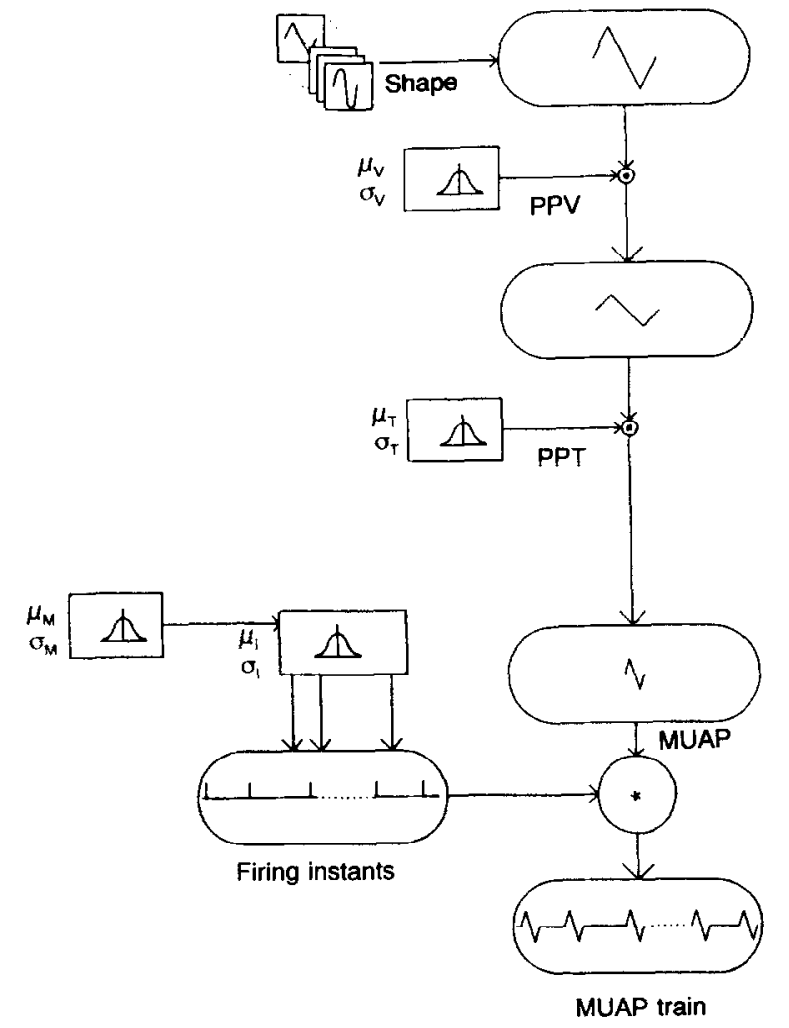

FIG. 1. Generation of one MUAP train. First a MUAP shape is chosen from a library. Then values for MUAP PPV and MUAP PPT are drawn from Gaussian distributions (mean $\mu_{V}$ resp. $\mu_{T}, S D \sigma_{V}$ resp. $\left.\sigma_{T}\right)$ and used to create an MUAP. An mIPI is drawn from a distribution function $\left(\mu_{M}, \sigma_{M}\right)$, representing the variability in mIPI among MUAP trains. The MIPI value is used as a mean value for the Gaussian distribution function $\left(\mu_{1}, \sigma_{1}\right)$ from which subsequent IPIs are drawn. Convolution of the MUAP with the pulse train results in an MUAP train.

sumed to be constant, its shape, amplitude, and duration not being changed during the simulation.

\section{Firing Patterns}

For each MUAP train a mean IPI (mIPI) was drawn from a Gaussian distribution $\left(\mu_{M}, \sigma_{M}\right)$. This distribution function expressed the variation in mIPI values among the different MUAP trains. As a next step the drawn mIPI was used as the mean value of a second distribution function $\left(\mu_{1}, \sigma_{1}\right)$. From this distribution function the subsequent IPIs for the MUAP train were drawn.

The IPI values were assumed to behave like independent samples of a Gaussian distribution $(22,23)$. According to experimental findings, a Gaussian distribution is justified (4) when the mean firing rate exceeds $\sim 10 \mathrm{~Hz}(21)$. 


\section{Synchronization}

To simulate synchronization, and thus study properties of $S f_{i j}$ in Eq. 4, the points of time at which MUAPs occur in MUAP trains were correlated. Each point of time within a synchronized MUAP train was drawn from a Gaussian distribution, placed symmetrically around the corresponding point of time in the first MUAP train (31) (Fig. 2). The choice of a Gaussian distribution was an approximation of the experimental findings and theoretical work of Kirkwood and Sears (11), who investigated changes in the state of depolarization of one motoneuron at the moment other motoneurons of the same pool were activated.

\section{Experiments}

Single MUAPs were extracted from the interference surface EMG patterns by means of a needletriggered averaging method (10). Bipolar surface electrodes (interelectrode distance $20 \mathrm{~mm}$ ) were placed on the $\mathrm{m}$. biceps brachii halfway between the motor endplate zone and the distal tendon, aligned in the direction of the muscle fibers. The subject was asked to exert a constant isometric force (range 10-40\% maximum voluntary contraction) and the needle electrode was inserted in the muscle between the two surface electrodes and manipulated at random until clear single-fiber action potentials (SFAPs) were found. The positive slope of the SFAPs was used to trigger the averaging process (window $100 \mathrm{~ms} ; 1,024$ samples).

The single-fiber signal was high-pass-filtered at $200 \mathrm{~Hz}$ and low-pass-filtered at $20 \mathrm{kHz}$. The surface electrode signal was high-pass-filtered at $10 \mathrm{~Hz}$ and low-pass-filtered at $1 \mathrm{kHz}$.

MUAPs were collected from three healthy subjects who all gave their informed consent to cooperate in this study. With each MUAP the PPT and

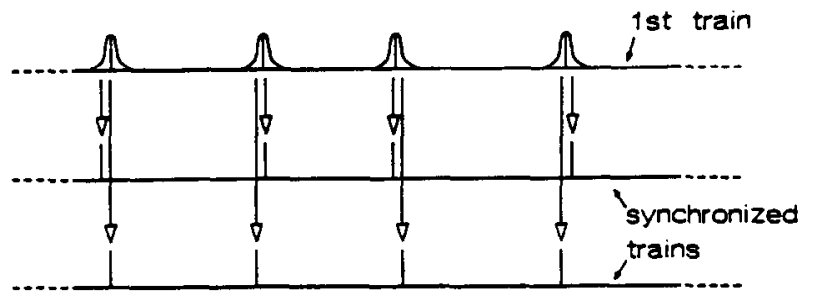

FIG. 2. The implementation of synchronization. The points of time MUAPs occur in synchronized MUAP trains are drawn from Gaussian distribution functions $\left(0, \sigma_{S}\right)$, placed symmetrically around the points of time MUAPs occur in the first MUAP train (31). the PPV were determined and then used to normalize the MUAP to obtain the MUAP shape.

With the same electrode placement, recordings were made of the interference EMG pattern, while the subject was asked to maintain a constant isometric force (range $20-40 \% \mathrm{MVC}$ ). The EMG record was sampled at $1,024 \mathrm{~Hz}$ during 1-s periods and transferred to a personal computer for data analysis.

\section{Analysis of EMG Patterns}

Each simulation generated records of 2,048 points, representing $2 \mathrm{~s}$ of EMG sampled with 1,024 $\mathrm{Hz}$. Both simulated EMG patterns and experimentally obtained recordings were analyzed by the same software (9). The power spectrum was calculated by means of a modified Blackman-Tukey algorithm.

The MF is the frequency dividing the EMG power spectrum $S e(f)$ into two parts of equal power:

$$
\int_{0}^{\mathrm{MF}} \operatorname{Se}(f) d f=\int_{\mathrm{MF}}^{\infty} \operatorname{Se}(f) d f=\frac{1}{2} \int_{0}^{\infty} \operatorname{Se}(f) d f
$$

\section{RESULTS}

\section{Unsynchronized Firing}

Figure 3 shows the relation between MF and the standard deviation of the IPI distribution function $\left(\sigma_{1}\right)$, reflecting the irregularity of the motor unit firing process. The figure shows that an increase of $\sigma_{I}$ slightly shifts MF upward. According to experimental findings (22), typical values of $\sigma_{\mathrm{I}}$ are between 5 and $15 \mathrm{~ms}\left(\mu_{\mathrm{I}}=100 \mathrm{~ms}\right)$. Within this range $\mathrm{MF}$ varies $<5 \%$.

The relation between $\mathrm{MF}$ and $\mu_{\mathrm{M}}$, the mean value of the distribution function that reflects the variation in MIPI among the MUAP trains, is shown in Fig. 4. Clearly, MF is hardly sensitive to variation of this parameter. When the mean firing rate of a pool of motor units is raised from 5 up to 20 pulses per second, MF will increase $\sim 5 \%$.

Variation of the third firing process parameter, the standard deviation of the mIPI distribution function $\left(\sigma_{M}\right)$, reflecting the variability in mIPI among the MUAP trains, did not affect MF.

\section{Synchronized Firing}

Figure 5 shows the relation between MF and the standard deviation of the distribution function $\left(\sigma_{S}\right)$ 


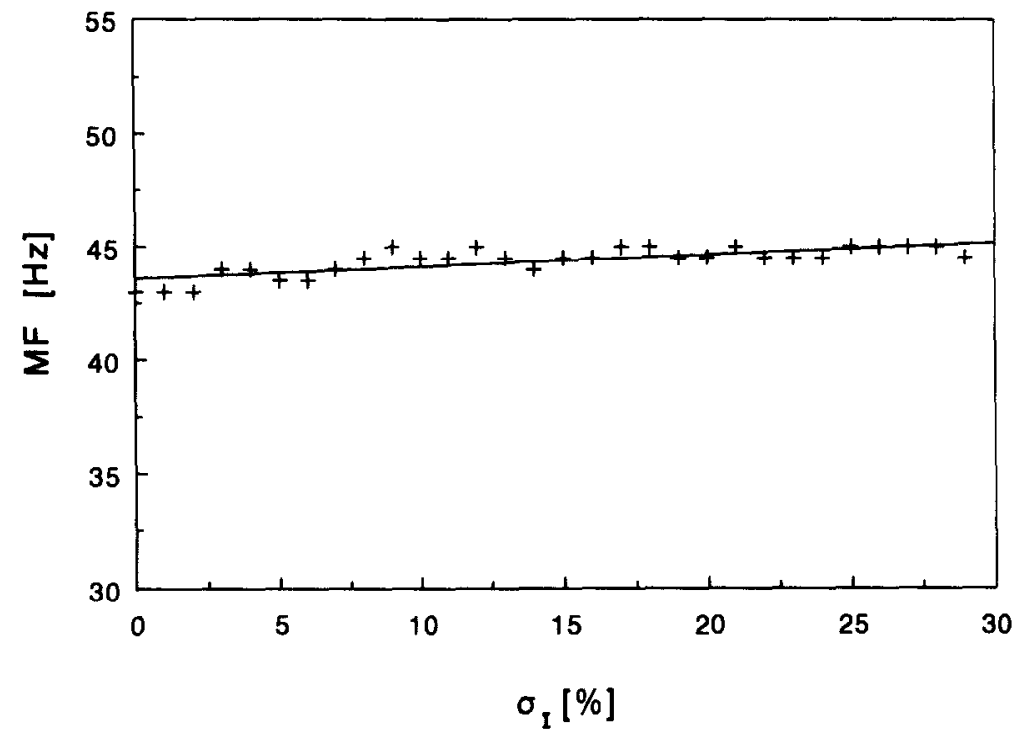

FIG. 3. The relation between MF and the standard deviation of the IPI distribution function $\left(\sigma_{1}\right)$ (simulation parameters: $N=1, \mu_{T}=10 \mathrm{~ms}$, $\sigma_{\mathrm{T}}=5 \%, \mu_{\mathrm{M}}=100 \mathrm{~ms}, \sigma_{\mathrm{M}}=0 \%, N_{\mathrm{S}}=0$ ). that links the firing instants in the different synchronized MUAP trains. This relation was calculated for different sizes of the subpopulation with synchronized activity $\left(N_{\mathrm{S}}\right)$.

When all MUAP trains were involved in synchronous firing $\left(N_{\mathrm{S}}=100 \%\right)$, an increase of $\sigma_{\mathrm{S}}$ from zero lowered MF substantially. However, above a certain value of $\sigma_{S}$, MF was found to increase again up to its original value. This means that in case of perfect synchronization, as during electrical stimulation of the muscle, MF has approximately the same value as without synchronization.

When only part of the motor unit pool was assumed to fire synchronously, not only the effect on MF was found to be less, but also the curve changed its shape. With lower values of $N_{\mathrm{S}}$, the minimum of MF was reached at lower values of $\sigma_{S}$.

\section{Sensitivity to MUAP Characteristics}

Figure 6 shows the relation between MF and the reciprocal value of the mean MUAP PPT $\left(\mu_{T}\right)$ for two different MUAP shapes. MF was found to be linearly related to the reciprocal value of $\mu_{T}$, the MUAP shape thus affecting only the slope of this relation. This finding made it possible to quantify an MUAP shape as the slope of this relation.

Figure 7 shows the relation between $\mathrm{MF}$ and the variability in MUAP PPT $\left(\sigma_{\mathrm{T}}\right)$ in the motor unit pool. Increasing $\sigma_{\mathrm{T}}$ caused a slight shift of MF to

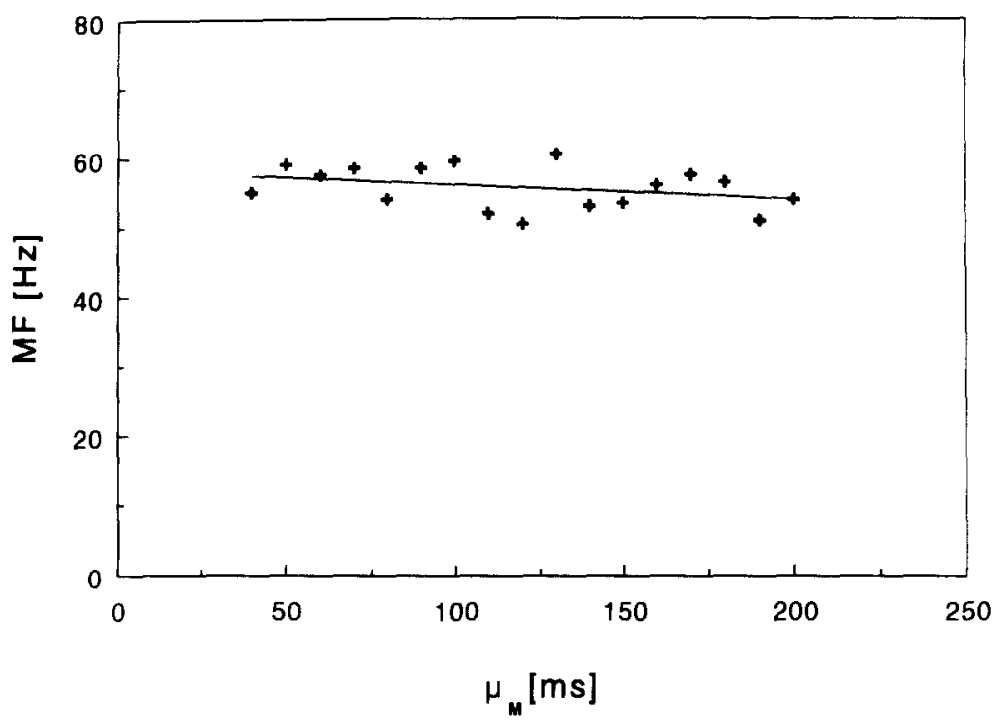

FIG. 4. The relation between MF and the mean of the mIPI distribution function $\left(\mu_{M}\right)$ (simulation parameters: $N=100, \mu_{T}=10 \mathrm{~ms}, \sigma_{T}=5 \%$, $\sigma_{M}=5 \%, \sigma_{1}=10 \%, N_{S}=0$ ) 


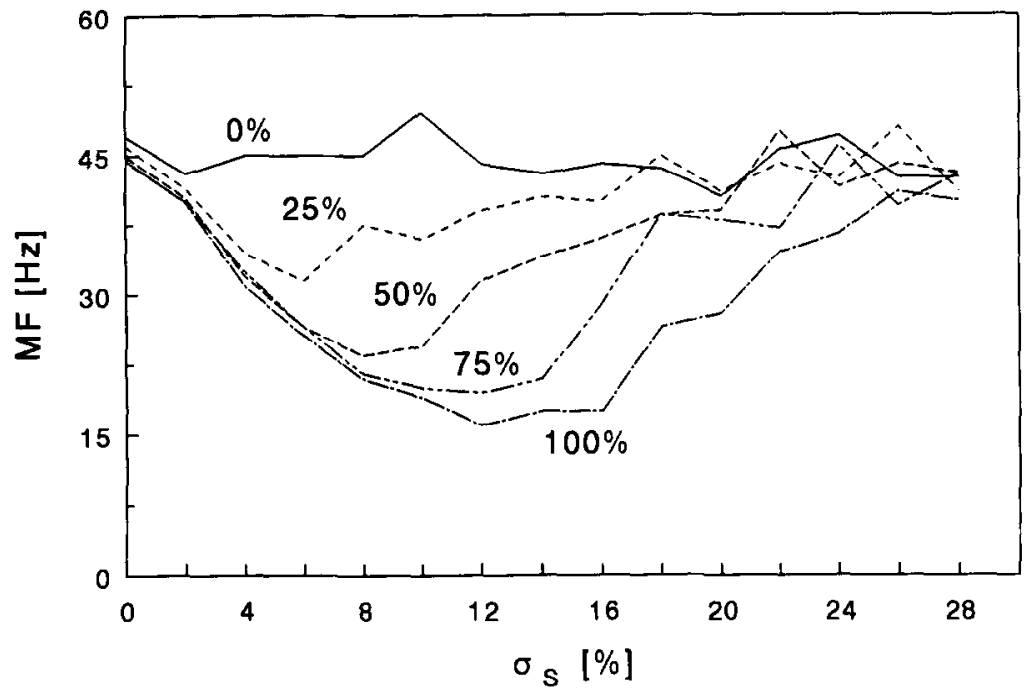

FIG. 5. The relation between MF and the standard deviation of the synchronization distribution function $\left(\sigma_{\mathrm{s}}\right)$, with different percentages of synchronously firing motor units $\left(N_{S}\right)$ (simulation parameters: $N=100, \mu_{\mathrm{T}}=10 \mathrm{~ms}, \sigma_{\mathrm{T}}=$ $10 \%, \mu_{M}=100 \mathrm{~ms}, \sigma_{M}=10 \%, \sigma_{1}=13 \%$ ). lower values. This shift was $\sim 10 \%$ for the whole range of $\sigma_{\mathrm{T}}\left(0,25 \% \mu_{\mathrm{T}}\right)$ used during simulation.

\section{Comparison Between Simulation Results and EMG Recordings}

MF values obtained from simulations, using experimental MUAPs, were compared with MF values directly calculated from EMG recordings. With each subject, the experimental MUAPs were converted to input data $\left(\mu_{T}, \sigma_{T}, \mu_{V}, \sigma_{V}\right.$, and MUAP shapes) for the model and the values of the other input parameters were chosen within physiological ranges $\left(N=100, \mu_{M}=100 \mathrm{~ms}, \sigma_{M}=20 \%, \sigma_{I}=\right.$ $13 \%)$. It was assumed that no synchronization $\left(N_{\mathrm{S}}\right.$ $=0$ ) occurred.
With each MUAP shape, the results of 30 simulations, using the individual MUAP statistics, were averaged to obtain one MF value. The relative width of the $95 \%$ confidence interval of these MF values was $5 \%$. The results are shown in the graphs of Fig. 8 as histograms of MF values.

An estimate of MF of the actual EMG recordings was obtained by averaging the MF values obtained from 10 recordings of each subject. The $95 \%$ confidence intervals of the mean MF values were calculated to compare the results. These are shown as bars in the graphs of Fig. 8. Assuming a correct estimation of all model parameters, including $\mu_{T}$ from the limited amount of MUAPs, the bar should be in the middle of the histogram (e.g., Fig. 8B). The results in Fig. 8 indicate that the estimated

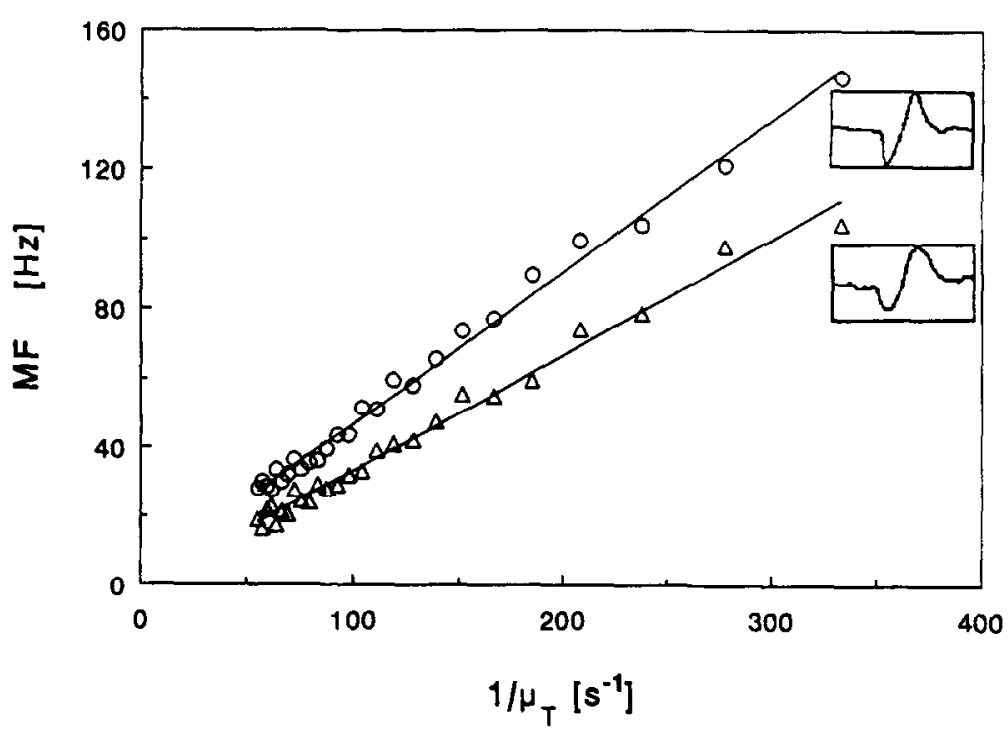

FIG. 6. The relation between MF and the reciprocal value of the mean of the MUAP PPT distribution $\left(\mu_{T}\right)$, with two different MUAP shapes (simulation parameters: $N=100, \sigma_{T}=10 \%, \mu_{M}$ $=100 \mathrm{~ms}, \sigma_{\mathrm{M}}=10 \%, \sigma_{1}=13 \%, N_{\mathrm{S}}=0$ ). 


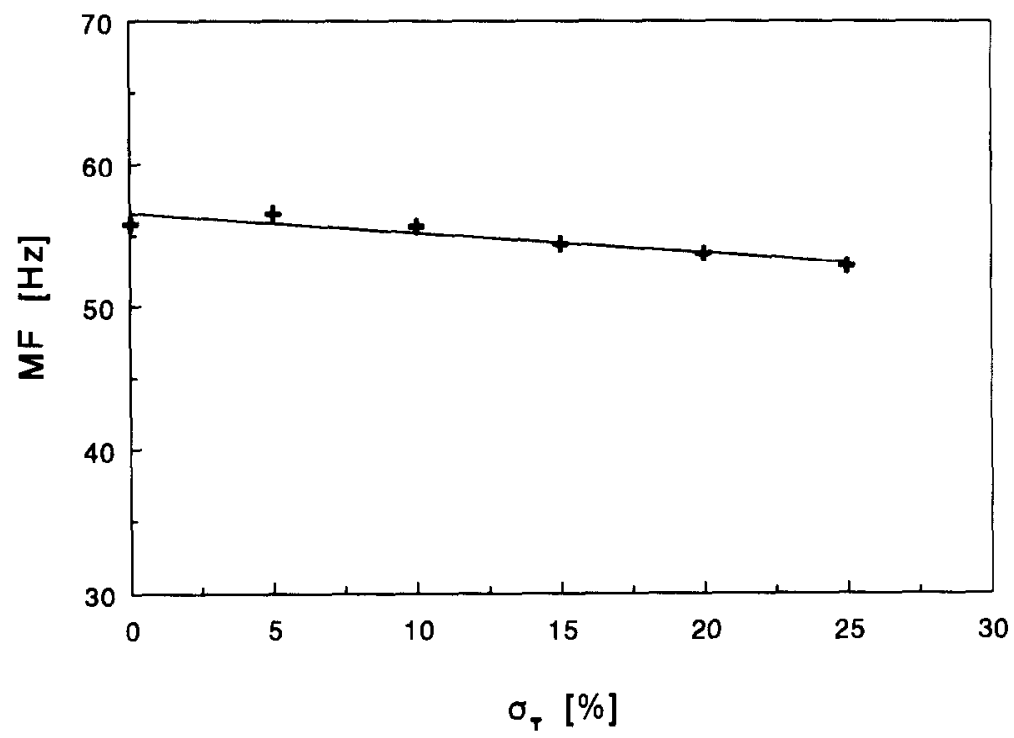

FIG. 7. The relation between MF and the standard deviation of the MUAP PPT distribution function $\left(\sigma_{T}\right)$ (simulation parameters: $N=100$, $\mu_{T}=8 \mathrm{~ms}, \mu_{M}=100 \mathrm{~ms}, \sigma_{M}=10 \%, \sigma_{1}=13 \%$, $\left.N_{\mathrm{S}}=0\right)$. value of MF from actual recordings is equal to or higher than predicted by simulation.

\section{Interindividual and Intraindividual Differences}

Considering the relations found between the model input parameters and MF, it can be predicted that, in absence of synchronization, variations in MF will be largely caused by variations in mean MUAP PPT $\left(\mu_{T}\right)$ and MUAP shape. To investigate the role of $\mu_{T}$ and MUAP shape in interindividual and intraindividual differences in MF, first the effect of a particular MUAP shape was defined as the slope $(S H)$ of the MF vs. $1 / \mu_{\mathrm{T}}$ relationship. Thus;

$$
S H=\mathrm{MF} \cdot \mu_{\mathrm{T}}
$$

It then follows from the results illustrated in Fig. 6, showing a linear relation with zero axis intercepts, that $S H$ does not depend on $\mu_{\mathrm{T}}$ and MF. Calculated $S H$ values of three subjects are shown in the histograms in Fig. 9.

As a next step, the relative standard deviations of the three individual distribution functions of PPT and $S H$ were calculated, regarding them as a measure of their relative variation. Also the standard deviations of all PPT and $S H$ values, pooled into one set, were calculated. The results are shown in Table 1. From this table it can be seen that within a muscle, MUAPs show a variation in both $S H$ and PPT. The ratio between the relative standard deviations in $S H$ and PPT show that the variability of PPT ranges between 1.00 and 1.78 times the variability of $S H$. Because MF is equally sensitive to relative variations in $S H$ and PPT, this indicates that both MUAP parameters contribute in a similar way to variations in MF.

The statistics concerning the whole set of measured MUAPs reveal some information on interindividual variations in $S H$ and PPT. For $S H$ the overall standard deviation is in the same range as the three individual standard deviation values. This indicates a large overlap of the individual distribution functions of the MUAP shape parameter. In contrast to this, the overall standard deviation for PPT is two to three times larger than the individual standard deviations, which indicates that these distribution functions do overlap only for a small part (Figs. 8 and 9). These findings were confirmed by the $p$ values resulting from two identical analysis of variance tests $(d f=2,21)$, one applied to the set of PPT data and one applied to the set of $S H$ data. The $\mathrm{p}$ value for the $S H$ data was $0.75>\mathrm{p}_{\mathrm{SH}}>0.5$ and that for the PPT data was $0.25>\mathrm{p}_{\mathrm{PPT}}>0.10$, indicating stronger evidence for interindividual differences in PPT than for interindividual differences in $S H$.

\section{DISCUSSION}

In comparison with previous models $(5,13,19,22$, $23,30,31$ ), a number of changes were made to make the model more realistic. These include the library of MUAP shapes and the use of distribution functions to account for the natural variability in mean firing rates, MUAP PPT, and MUAP peak-peak amplitude. The model input parameters were not cor- 

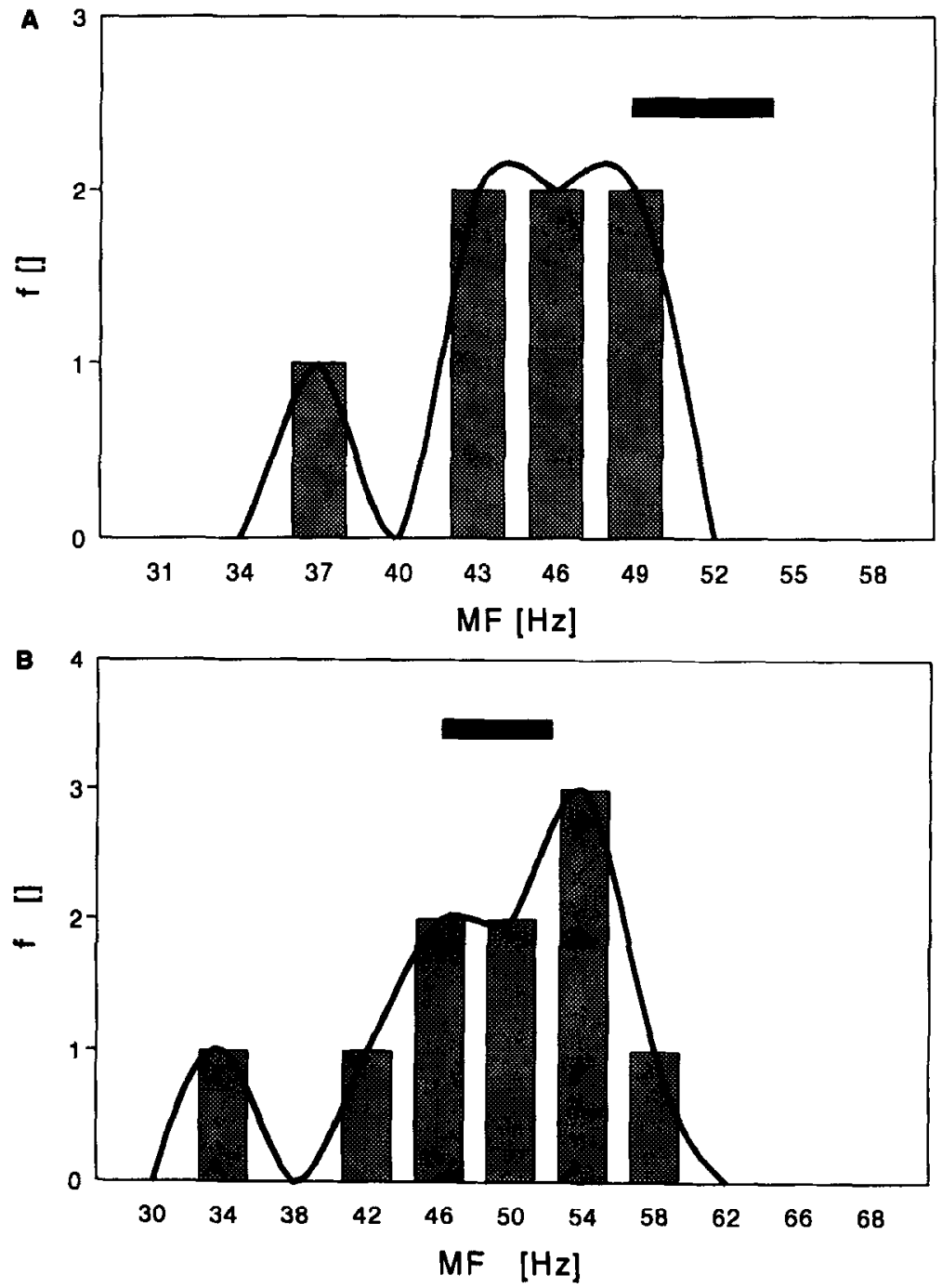

FIG. 8. Histograms of MF values obtained by simulation. For each MF value, an experimental MUAP shape was used as input. Pooled, individual MUAP data were used to estimate the PPT parameters (simulation parameters: $N=100$, $\mu_{M}=100 \mathrm{~ms}, \sigma_{M}=20 \%, \sigma_{1}=13 \%, N_{s}=0$ ). The $95 \%$ confidence interval of a mean MF, calculated from MF values of 10 experimental EMG recordings in the same subject, is shown as a horizontal bar. The curve shows a best fit with a second-order spline. A-C: Data of, respectively, subjects $A, B$, and $C$.

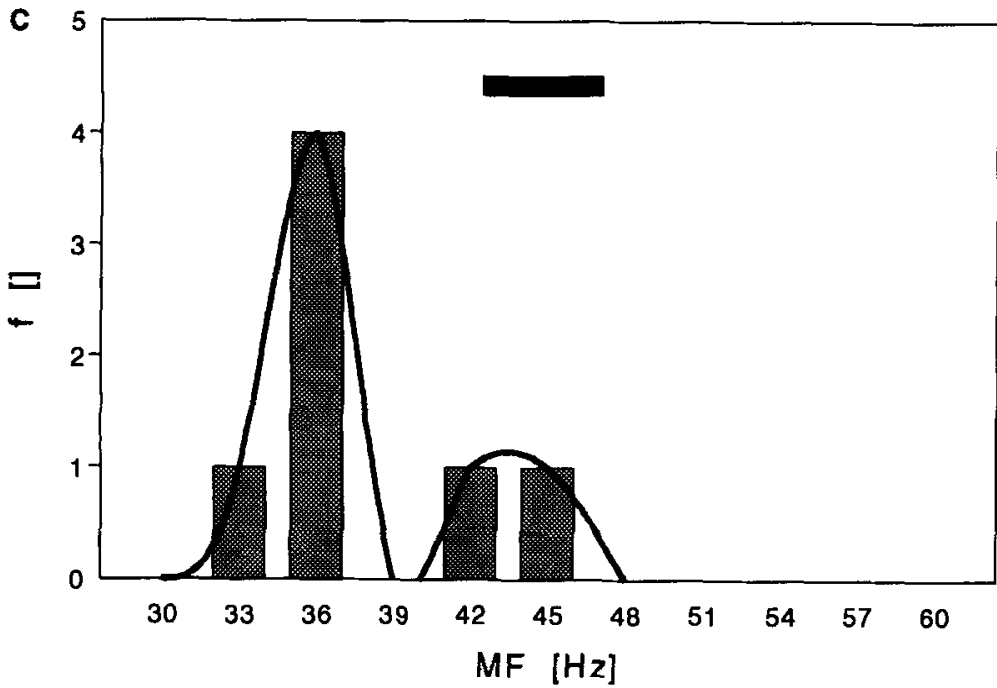




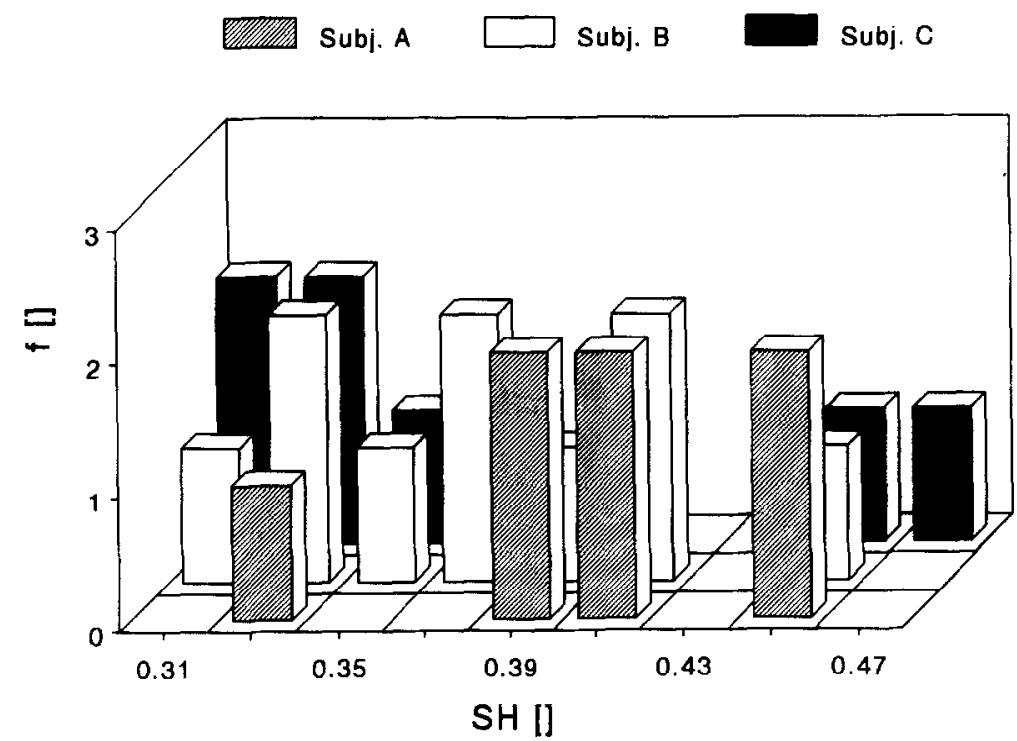

FIG. 9. Histograms of the MUAP shape parameter $(\mathrm{SH})$, reflecting the influence of the MUAP shape on the MF (defined in Eq. 6). Values calculated from experimental MUAP data in three subjects.

related because the nature of such correlation is largely unknown. In addition, it is relatively simple to predict the effect of a possible correlation from the predicted effect of variation of isolated parameters.

The results obtained by simulation clearly predict that variability in MF is caused by variations in MUAP shape $(S H)$, mean MUAP PPT $\left(\mu_{T}\right)$, and synchronization $\left(N_{\mathrm{S}}\right.$ and $\left.\sigma_{\mathrm{S}}\right)$. This is in agreement with findings of Person and Libkind (22). They computed the dominating frequency (frequency of maximum power density) to be linearly related to the reciprocal value of the duration of their artificial MUAP shape. The ratio between the duration of their MUAP shape and the mean PPT is constant, while also their dominating frequency is comparable to MF owing to the rather symmetrical shape of their power spectrum. This result, however, seems

TABLE 1. Mean values of MUAP PPT, relative standard deviations of MUAP PPT (dPPT/PPT), relative standard deviations of MUAP shape parameter (dSH/SH), calculated from experimental MUAP data in three subjects, and relative standard deviations of all $P P T$ and $\mathrm{SH}$ values pooled into one set

\begin{tabular}{lccc}
\hline & $\begin{array}{c}\text { PPT } \\
(\mathrm{ms})\end{array}$ & $\begin{array}{c}d \text { PPT/PPT } \\
(\%)\end{array}$ & $\begin{array}{c}d S H / S H \\
(\%)\end{array}$ \\
\hline Subject A & 8.9 & 13.0 & 9.6 \\
Subject B & 7.6 & 15.1 & 8.5 \\
Subject C & 9.6 & 14.7 & 14.7 \\
Pooled A + B + C & - & 37.1 & 12.2 \\
\hline
\end{tabular}

at variance with the observations of Tanzi and Taglietti (27). They found a linear relation (negative slope) between the estimated duration of experimentally obtained MUAPs and the frequency of maximal power density. The seeming difference with our results may be only apparent and due to their limited number of samples (9), which allowed them to fit their data with a linear curve instead of with a $1 / x$ relation.

Our results predict that MF is hardly sensitive to variation of the firing process parameters. This indicates that variations in $S f_{i i}$ have little influence in the first term on the right of Eq. 4. Apparently, despite overlap of the power spectra of the MUAPs and the firing processes, the power density of the MUAP spectra is low at those frequencies where peaks occur in the spectra of the firing processes.

From the figure given by Pan et al. (19), it can be derived that MF increases as little as $5 \mathrm{~Hz}$ while the mean firing rate is varied from 10 to $50 \mathrm{~Hz}$. This shift is even less than indicated by our results, probably owing to the higher value of the MUAP PPT they used during simulation.

We also varied the other part of the first term in Eq. 4 , which is the function $\left[M_{i}(\omega)\right]$ that describes the MUAP properties. When we regard the variation of $1 / \mu_{\mathrm{T}}$ as a compression/expansion of the MUAP power spectrum along the frequency axis, a linear relation over such a wide range as shown in Fig. 6 could be obtained only when the power spectrum of the firing process was rather flat in the area involved. 
The analysis of the experimentally obtained MUAPs indicated that within one muscle differences in PPT and MUAP shape $(S H)$ are present, both contributing to variations in MF. Such differences may result in a correlation between MF and the level of contraction if one or both parameters are related to the recruitment level of motor units. Solomonow et al. (26) found a linear increase of MF of the M-wave with stimulation level (normal recruitment order). Also in experimental studies in humans, a relation between force level and MF has been observed $(3,12)$.

The analysis of interindividual differences in MF values indicated that these are caused primarily by differences in mean PPT $\left(\mu_{T}\right)$ rather than by differences in MUAP shape $(S H)$. The relatively low variability in $S H$ may be explained by considering that the MUAP shape changes especially when the electrodes are moved along the direction of the muscle fibers (25). This variability was diminished during the experiments by placement of the electrodes halfway between the distal tendon and the motor endplate zone, a region where the MUAP shape is relatively constant.

The other MUAP parameter, the PPT, is determined by factors like muscle fiber conduction velocity, the spatial dispersion of the endplates, and anatomical properties of the muscle (14). These factors are merely dependent on the subject rather than on the recording method. This seems in agreement with experimental findings of Broman et al. (3), who observed intersubject differences in MF, which correlated with subject muscle fiber conduction velocity and limb circumference.

The use of experimental MUAPs as input for the model resulted in lower or equal values of MF in comparison with the confidence interval of MF obtained from EMG recordings in the same subject. Differences might be due to the limited amount of MUAPs $(7,7$, and 10$)$ used to estimate the mean PPT in each subject. Another cause could be an underrepresentation of MUAPs with a low PPT, probably belonging to motor units with higher recruitment levels. When the needle was manipulated, subjects often tended to lower the force level to avoid pain.

The results of this study indicate clearly which parameters at the motor unit level contribute significantly to changes in MF and which parameters do not. Two of the relevant parameters, MUAP shape and mean PPT, are well understood in terms of the underlying physiological processes. Synchroniza- tion was predicted to be able to decrease MF considerably, depending on the number of motor units involved $\left(N_{\mathrm{S}}\right)$ and the spread in the firing instants $\left(\sigma_{S}\right)$. MF was predicted to be equal for EMG signals with perfect $\left(\sigma_{S}=0\right)$ and with no synchronized motor unit activity. This means that the $\mathrm{M}$-wave equals the mean MUAP with respect to shape and PPT. This prediction coincides with experimental finding of Knaflitz et al. (12), as their figures do not show systematic differences in MFs obtained during voluntary and electrically elicited contractions.

A broadly accepted theory on the mechanism of synchronization as well as on its role during voluntary contractions is missing. In a number of studies $(8,16)$, it has been shown that motor units tend to fire synchronously more often than can be expected from coincidence. In other studies, the existence of synchronization was questioned (28). Synchronization is also mentioned to explain part of the shift of the power spectrum toward lower frequencies as observed in muscle fatigue $(3,18,32)$.

Part of the differences in literature may be explained by considering $N_{\mathrm{S}}$, the number of motor units involved in synchronization, as a statistical time-varying parameter. It then describes a population of motor units that may change constantly, but with an average size equal to $N_{\mathrm{S}}$. As a consequence, the effect of synchronization will be observed always in the surface EMG signal, but it may be missed or underestimated easily when only a small number of motor units is observed.

\section{REFERENCES}

1. Blinowska A, Verroust J, Cannet G: An analysis of synchronization and double discharge effects on low frequency electromyographic power spectra. Electromyogr Clin Neurophysiol 20:465-480, 1980.

2. Boukes R: Force and surface EMG measurements in patients with neuromuscular disorders. MD thesis, Vrije Universiteit Amsterdam, Krips Repro Meppel, Amsterdam. 1982.

3. Broman H, Bilotto G, De Luca CJ: Myoelectric signal conduction velocity and spectral parameters: influence of force and time. J Appl Physiol 58:1428-1437, 1985.

4. Clamann HP: Statistical analysis of motor unit firing patterns in a human skeletal muscle. Biophys $J$ 9:1233-1251, 1969.

5. De Luca CJ: Physiology and mathematics of myoelectric signals. IEEE Trans Biomed Eng 26:313-325, 1979.

6. De Luca CJ: Myoelectrical manifestations of localized muscular fatigue in humans. CRC Crit Rev Biomed Eng 11:251279, 1984.

7. De Luca CJ and Forrest WJ: Some properties of motor unit action potential trains recorded during constant force isometric contractions in man. Kybernetik 12:160-168, 1973.

8. Dietz V, Bischofberger E, Wita C, Freund HJ: Correlation between the discharges of two simultaneously recorded mo- 
tor units and physiological tremor. Electroencephalogr Clin Neurophysiol 40:97-105, 1976.

9. Hermens HJ, Boon KL, Zilvold G: The clinical use of surface EMG. Electromyogr Clin Neurophysiol 24:243-265, 1984.

10. Hermens HJ, van Bruggen TAM, Rutten WLC, Wilts G: A stochastic model for simulation of surface EMG patterns. In: Electrophysiological Kinesiology. International Congress Series 804, Elseviers Science Publishers, Amsterdam, pp 231-234, 1988

11. Kirkwood PA and Sears TA: The synaptic conncxions to intercostal motoneurones as revealed by the average common excitation potential. J Physiol 275:103-134, 1978.

12. Knaflitz M, Merletti R, De Luca CJ: Inference of motor unit recruitment order in voluntary and electrically elicited contractions. J Appl Physiol 68:1657-1667, 1990.

13. Lago PJ and Jones NB: Effect of motor unit firing time statistics on EMG spectra. Med Biol Eng Comput 15:648-655, 1977.

14. Lindstrom LH and Magnusson RI: Interpretation of myoelectric power spectra: a model and its applications. IEEE Trans Biomed Eng 65:653-662, 1977.

15. Merletti R, Biey D, Biey M, Prato G, Orusa A: On-line monitoring of the median frequency of the surface EMG power spectrum. IEEE Trans Biomed Eng 32:1-7, 1985.

16. Milner-Brown HS, Stein RB, Lee RG: Synchronization of human motor units: possible roles of exercise and supraspinal reflexes. Electroencephalogr Clin Neurophysiol 38:245254. 1975.

17. Muro M, Nagata A, Murakami K, Moritani T: Surface EMG power spectral analysis of neuromuscular disorders during isometric and isotonic contractions. Am J Phys Med 61:244 $254,1982$.

18. Naeije M, Zorn H: Relation between EMG power spectrum shifts and muscle fibre action potential conduction velocity changes during local muscular fatigue in man. Eur $J$ Appl Physiol 50:23-33, 1982.

19. Pan ZS, Zhang Y, Parker PA: Motor unit power spectrum and firing rate. Med Biol Eng Comput 27:14-18, 1989.

20. Papoulis A: Probability, Random Variables, and Stochastic Processes. McGraw-Hill, Kogakusha, 1965.

21. Person RS and Kudina LP: Discharge frequency and discharge pattern in human motor units during voluntary con- tractions of muscle. Electroencephalogr Clin Neurophysiol 32:471-483, 1972.

22. Person RS and Libkind MS: Simulation of electromyograms showing interference patterns. Electroencephalogr Clin Neurophysiol 28:625-632, 1970.

23. Piotrkiewicz M: A study of surface electromyograms by means of digital simulation. Electromyogr Clin Neurophysiol 18:83-94, 1978.

24. Roy SH, De Luca CJ, Casavant DA, Emley MS: EMG assessment of muscular deficits associated with chronic low back pain. In: Electrophysiological Kinesiology, International Congress Series 804, Elseviers Science Publishers, Amsterdam, pp 451-454, 1988.

25. Sollie G, Hermens HJ, Boon KL, Wallinga de Jonge W, Zilvold G: The boundary conditions for measurement of the conduction velocity of muscle fibres with surface EMG Electromyogr Clin Neurophysiol 25:45-56, 1985.

26. Solomonow M, Baten CTM, Smit J, Baratta R, Hermens HJ, D'Ambrosia R, Shoji H: Electromyogram power spectra frequencies associated with motor unit recruitment strategies. $J$ Appl Physiol 68:1177-1185, 1990.

27. Tanzi $F$ and Taglietti V: Spectral analysis of surface motor unit action potentials and surface interference electromyogram. IEEE Trans Biomed Eng 28:318-324, 1981.

28. Taylor A: The significance of grouping of motor unit activity. J Physiol 162:259-269, 1962.

29. Ten Hoopen M: Examples of power spectra of uni-variate point processes. Electroencephalogr Clin Neurophysiol 70:68-72, 1974.

30. Weytjens JLF and van Steenberghe D: Spectral analysis of the surface electromyogram as a tool for studying rate modulation: a comparison between theory, simulation, and experiment. Biol Cybern 50:95-103, 1984.

31. Weytjens JLF and van Steenberghe D: The effects of motor unit synchronisation on the power spectrum of the electromyogram. Biol Cybern 51:71-77, 1984.

32. Zwarts MJ, van Weerden TW, Haenen HTM: Relationship between average muscle fibre conduction velocity and EMG power spectra during isometric contraction, recovery and applied ischemia. Eur J Appl Physiol 56:212-216, 1987.

33. Zwarts MJ, van Weerden TW, Links TP, Haenen HTM Oosterhuis HJGH: The muscle fibre conduction velocity and power spectrum in familial hypokalemic periodic paralysis. Muscle Nerve 11:166-173, 1988 